receipt and care-provision by the older population as maintaining these activities could be challenging with travel restrictions and recommended physical distancing. This study aimed to determine if prevalence of informal caring changed during the COVID-19 pandemic, and examine longitudinal trends in quality of life, stress, and depressive symptoms in carers.

Methods We analysed the COVID-19 self-completion questionnaire (SCQ) sub-study (June-November 2020) of The Irish Longitudinal Study on Ageing (TILDA), a nationally representative study of people aged $\geq 50$ years ongoing since 2009 . A total of 3,043 participants aged $\geq 60$ years were included. Participants were asked if they cared for someone during the COVID-19 pandemic, their relationship to the recipient, and number of hours/week. Outcome measures collected were CES-D8, Perceived stress Scale (PSS) and CASP12. Change in prevalence of caring from previous waves was examined. Multi-level regression analyses examined longitudinal trends in CED-D8, PSS and CASP12 scores by caring status since Wave 3 (2014), adjusting for sociodemographic variables. Analyses were conducted in Stata 14.

Results Prevalence of informal caring tripled during the COVID-19 pandemic (15\% compared to 5\% in 2018). While $26 \%$ of carers reported they had stopped caring since the pandemic, most of those providing care were new carers (68\% of men and $66 \%$ of women carers). Becoming a new carer during the pandemic was associated with lower CASP12 scores $(\beta=-0.582,95 \%$ CI $-1.018,-0.146)$ and higher PSS ( $\beta=0.541,95 \%$ CI $0.316,0.765)$ compared to no caring. Caring for $\geq 50$ hours in the past week was associated with lower CASP12 $(\beta=-0.878,95 \%$ CI $-1.345,-0.412)$, higher PSS scores $(\beta=0.383$, 95\%CI $0.066,0.700)$ and higher depressive symptoms $(\beta=0.371,95 \%$ CI $0.251,0.490)$. This association was moderated by carer status; new carers with increased hours had higher CES-D8 compared to non-carers, with no difference for those who stopped or continued caring.

Discussion This study demonstrated that the reduction of availability of both formal and informal care for older people during the COVID-19 pandemic, led to increased caring by older household members and was associated with lower wellbeing and mental health. This study provides further evidence of the detrimental indirect effects of the pandemic for older people and emphasizes for policymakers the importance of prioritising formal and informal care arrangements for the older population.

\section{OP62 CARING IN THE TIME OF COVID-19, LONGITUDINAL TRENDS IN WELLBEING AND MENTAL HEALTH IN CARERS IN IRELAND: EVIDENCE FROM THE IRISH LONGITUDINAL STUDY ON AGEING (TILDA)*}

${ }^{1}$ Christine McGarrigle*, ${ }^{1}$ Mark Ward, ${ }^{1}$ Aisling O'Halloran, ${ }^{1}$ Celine Delooze, ${ }^{1,2}$ Rose Anne Kenny. 'The Irish Longitudinal Study on Ageing (TILDA), Trinity College Dublin, Dublin, Ireland; ' ${ }^{2}$ Mercer's Institute for Successful Ageing, St James's hospital, Dublin, Ireland

\subsection{6/jech-2021-SSMabstracts.62}

Background The initial public health response to the COVID19 pandemic in Ireland recommended that older people aged $\geq 70$ years remain at home, and physically isolate from those outside their household. This may have affected both care- 